\title{
DETECTING TRAFFIC CONDITIONS MODEL BASED ON CLUSTERING NODES SITUATIONS IN VANET
}

\author{
Ayman Abufanas and Evtim Peytchev \\ School of Science and Technologyy \\ Nottingham Trent University \\ Clifton Lane, Nottingham, NG11 8NS, UK \\ E.mail: \{ayman.abufanas2009, evtim.peytchev\}@ntu.ac.uk
}

\section{KEYWORDS}

Vehicular Ad-Hoc Network; Traffic Condition Detection; Clustering; Network Simulation; ITS; NS-3; Mobile Programming.

\begin{abstract}
In the last decade, cooperative vehicular network has been one of the most studied areas for developing the intelligent transportation systems (ITS). It is considered as an important approach to share the periodic traffic situations over vehicular ad hoc networks (VANETs) to improve efficiency and safety over the road. However, there are a number of issues in exchanging traffic data over high mobility of VANET, such as broadcast storms, hidden nodes and network instability.

This paper proposes a new model to detect the traffic conditions using clustering traffic situations that are gathered from the nodes (vehicles) in VANET. The model designs new principles of multi-level clustering to detect the traffic condition for road users. Our model (a) divides the situations of vehicles into clusters, (b) designs a set of metrics to get the correlations among vehicles and (c) detects the traffic condition in certain areas. These metrics are simulated using the network simulator environment (NS-3) to study the effectiveness of the model.
\end{abstract}

\section{INTRODUCTION}

$\mathrm{V}^{\mathrm{s}}$ EHICULAR ad hoc network is considered as new technology that has recently developed in terms of improving road safety, traffic knowledge and tourist information service. The issues of traffic control and management have become significantly important in order to utilize full capacity of all roads. In addition, the lack consideration of travel safety may also increase number of traffic accidents. For instance, approximately six million accidents are recorded annually in the United States alone and the same study shows that in the 2007, thousands of people have died and were injured in China due to cars crashes (Zhou et al. 2010).

Therefore, much effort has been placed to develop ITS applications to reduce the problems to avoid traffic jams or dangerous areas on their current routes. For instance, Bishop (2005) states that the combination of ITS and telecommunication systems and transport information systems is able to provide reliable forecast information to travellers to enhance safety and efficiency of the routes. These efforts are under-utilised so far in term of traffic information systems where every vehicle can be presented as a single node in an ad-hoc network (Thomas et al. 2004).

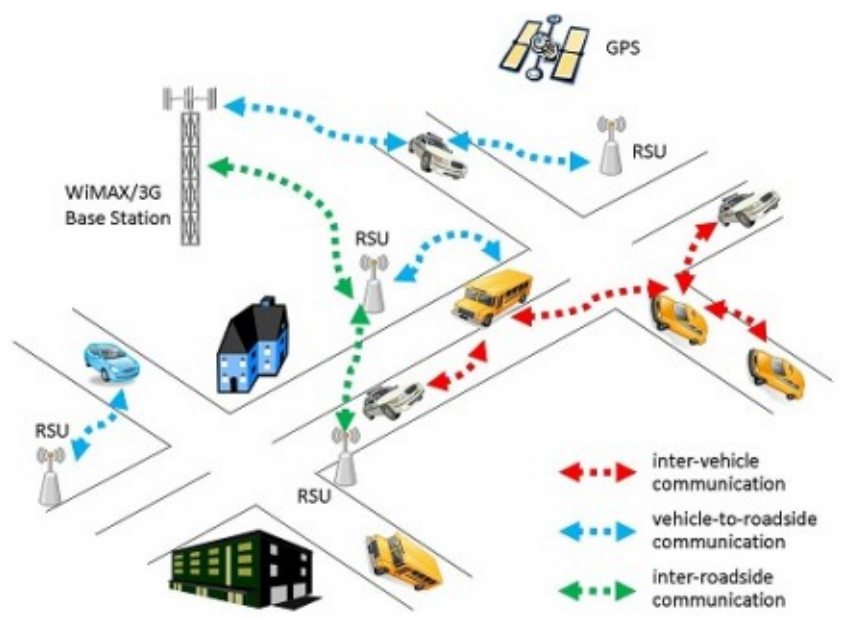

Figure 1: VANET concept (Khan 2010)

In fact, several recent relevant ITS applications in the current time require fixed infrastructure. Particularly, the activity of information exchange between vehicles and infrastructure is defined as Vehicle-to-Infrastructure (V2I) communications. Although, it provides several solutions for any transport challenges, it is arguable that V2I architecture has faced several restrictions such as the cost of sufficient infrastructure and reliable connection (Thomas et al. 2004).

According to these circumstances, researchers and commercial projects tend to focus on a different architecture of ITS applications, known as the vehicle-tovehicle (V2V) communications, which focus on direct message delivery between nodes (vehicles). Figure 1 illustrates the architecture of vanet including these types of communications. Currently, VANET-based wireless networks are a natural fit to intelligent information transportation system (ITS).

The rest of paper is structured as follows. Section II discusses related work of the proposed model. Section 
III demonstrates our model and methodologies that are applied. Then section IV generally concludes the paper and briefly introduces the future work of the project.

\section{RELATED WORK}

Designing traffic monitoring dissemination approach is still one of the open research challenges being investigated in VANET. Researchers often consider that it is significant to detect the specific traffic conditions over roads or junctions in vehicular networks (Praba \& Ranichitra 2013). On one hand, it is widely known that the more vehicles are on the road, more reliable and faster for information delivery in vehicular networks. On the other hand, the higher the density of vehicles, the more probability that traffic congestion or accidents may happen in the area. Therefore, the number of vehicles are on the road is a double-edged sword in order to achieve a high level of stability of networks.

The big challenge for reliable and fast data delivery is blind vehicles. Floating car data is a technology that gathers road status data from vehicles. FLOAT is a system of driving alongside the sections and recording the traffic conditions when road users travel (Bishop 2005). This scheme assumes that cars can be considered as a moving sensor that runs on a network. In addition, floating car data technology (FCD) has become widely used in many algorithms for solving the challenges of fixed monitoring systems (Li et al. 2012). Thus traffic information of FCD may improve drivers decisions for their route choices.

Manvi et al. (2009) introduced a cognitive model which performs pull and push processes on relevant and important data collected in VANETs and also they proposed a critical data retrieve agent called CIPLA. Sometimes, vehicles are interested to recollect latest weather information from other vehicles, such as fog, rain, etc. However, CIPLA seems to be complex and messages that forwarded by this system are also sent slowly, consequently, the delay may tend to be bigger and the performance might be low.

Vision car detection based system was presented for FCD, which helps to improve the context of Vehicular networks (Llorca et al. 2010). Packets are transmitted based on GPRS/UMTS communication which also uses a central unit to mix the expanded FCD. However, GPRS/UMTS is responsible for the delivering the packets, which it means that their model depends on an existed infrastructure such as stations, road side units (RSU) etc. Therefore, Carry messages then forward is quite appropriate mode with low mobility and acceptable delay networks (Li et al. 2012).

Therefore, Zhao \& Cao (2008) focused on V2V communication and they introduced the idea of "carrying then forwarding" packets, which means that packets will be temporarily kept in node's buffer until it obtains a connection with other node that enters the network.
Then the message will be forwarded to that node. They proposed vehicles assisted data delivery algorithms (VADD) which use dense roads to deliver the packet to obtain faster delivery. However, the authors didnt focus on a method of detecting the traffic situation whether it is dense or not.

Gamati et al. (2011) proposed algorithm for traffic detection, named (TCDA) in V2V communication. Their idea is based on using the optimum number of messages that provide sufficient indication to detect the conditions about the roads. They obtain good result compared with Flooding and AODV protocol. A serious question with TCDA model, however, is the idea of optimum number could be varied depending on weather and traffic situation, such as fog, snow, etc. They obtained good results compared with flooding protocol. Moreover, the algorithm did not clearly consider the issue of the boundaries of area that is covered to estimate the traffic condition. Moreover, other unconsidered parameters may affect on the reliability of detecting the conditions, such as speed, direction.

The proposed model is designed to take a place on an application layer in VANET. Therefore, the existing routing protocols are applied for exchanging and delivering packets among nodes in the network. It was decided to use Ahoc On Demand Distance Vector routing protocol (AODV) to achieve the routing suite in the model.

AODV is considered as a reactive routing protocol that enables dynamic multi-hop routing among participating nodes to establish and maintain a network (Perkins et al. 2003). AODV can manage low and relatively high mobility of nodes, as well as a variety of data traffic levels. Route Requests (RREQs), Route Replies (RREPs), Route Errors (RERRs), and hello packets are some types of the message defined by the protocol (Perkins et al. 2003). As it is found that AODV is suited sufficiently for VANET, authors decided to enhance AODV by adding traffic monitoring features.

The model combines routing functionality for delivery the messages to provide efficient detection of traffic situation; cluster aggregation of nodes to improve stability, avoid broadcast storms and finally a part of a system to define cloud information network in each cretin area. Our model (a) divides the situations of vehicles into groups, (b) designs a set of metrics to get the correlations among vehicles and (c) detects the traffic condition in certain areas.

\section{PROPOSED WORK}

Designing a reliable traffic detection model is a challenge, an interesting and widely considered by researchers. According to relevant works, previous models do not apply the idea of using clustered vehicles' situations to detect traffic conditions. The new model 
will consider this idea to achieve the aim of traffic condition detection over traffic scenario in ad hoc network. A framework of reliable traffic detection is designed and simulated in NS-3. IEEE 1609.4 and IEEE $802.11 \mathrm{p}$ standard is applied in the model for the communication between $\mathrm{V} 2 \mathrm{~V}$ and $\mathrm{V} 2 \mathrm{I}$ in our model. Moreover, the model will link the area between VANET environment and vehicle's driver decision based on the gathered messages from traffic roads status.

Figure 2 illustrates a general diagram of proposed work which includes two main phases as follow; First, mobility scenario, which is presented as generated mobility patterns by simulation of urban mobility software (SUMO), and AODV routing protocol for delivering packets between nodes. Second, phase is for traffic condition detection, it consists of (a) stage of augmenting and defining traffic condition into the mobility pattens file and (b) detecting the traffic condition stage by clustering the gathered messages. These messages reflect individual cars' situations, such as ABS (on/off), brake lights (on/off), windscreen wipers (on/off), etc.

\section{Generating Traffic Mobility Using SUMO}

SUMO is one of the most popular microscopic opensource tools for handling and simulating traffic in large scale of street networks (Krajzewicz et al. 2012). SUMO uses a number of tools to integrate roads network processing and traffic generation. The roads are represented as cretin edges of an oriented graph, with nodes being the intersections (SUMO 2015). In addition, Edges can carry relevant traffic information, such as street category, traffic lights location, speed limitations and other signs, etc.. Moreover, SUMO has a tool called net-convert, which is capable to convert digital maps (e.g., OpenStreetMap) into SUMO map format (SUMO 2015).

The algorithm 1 shows pseudocode of the suite of clustering and detecting the traffic condition in the proposed model. Figure 3 explains the stages of algorithm how they are simulated by presenting the levels of the model. The lowest level is to identify the network by the existing routing protocol. Then the traffic detection suite which includes (a) level of gathering vehicles' messages, and (b) clustering the vehicles based on the gathered traffic situations. Finally detecting the road conditions, including detecting clusters boundaries, based on studying the correlation level between cars' situations. In addition, the following equations explain the mathematical background for this aim.

Where messages $v 1, v 2, v 3, \ldots$ are the gathered individual situations, and $A, B, C, D, \ldots$ represent sets of traffic situations. Each set of vehicles will create their own cluster as long as they send the same type of messages, even these vehicles might not be physically neighbours.

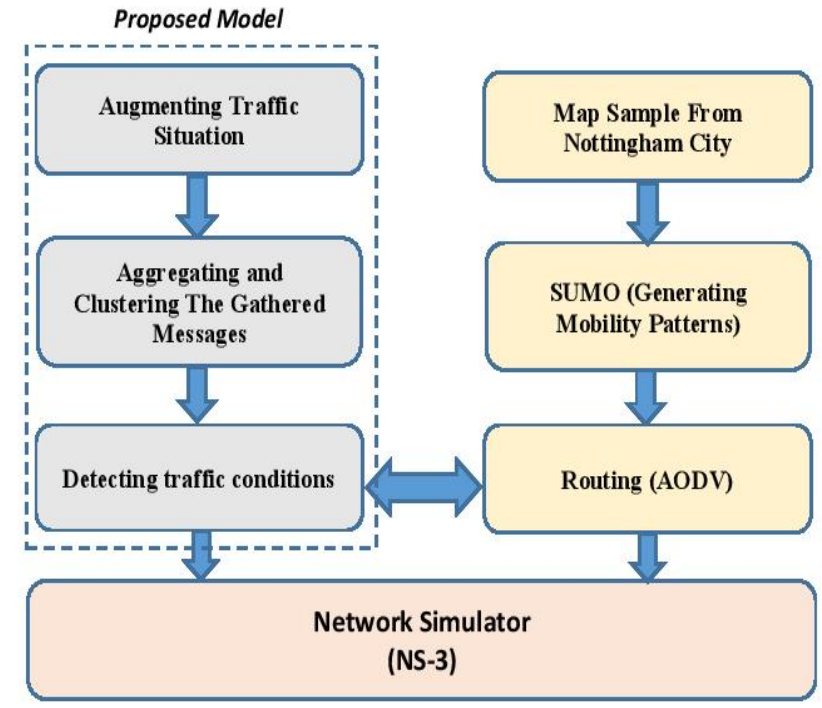

Figure 2: Proposed Framework of Traffic Condition Detection

initialization: number of vehicles is $n$, defining

traffic situations, $t$;

while within simulation time do

read message $v_{i}$;

if do messages have the same type of traffic situation then

if the type of message is already aggregated then

add to cluster $s_{i}$;

else

| keep this message

end

else

update the clusters by these messages;

end

while there is a correlation among $s_{n}$ do

if the correlation between cluster $s_{i}$ and $s_{j}$ is positive then

merge $s_{i}$ and $s_{j}$ into a new cluster $C_{i}$; else

I check next clusters

end

end

end

Algorithm 1: Pseudocode of The Algorithm

$$
\begin{gathered}
A=\{v 1, v 3, v 4, v 6\} \\
B=\{v 1, v 2, v 3, v 6\} \\
C=\{v 3, v 7, v 8\} \\
D=\{v 7, v 10\}
\end{gathered}
$$

After creating clusters of traffic situation, the model studies the correlation between them. As it is shown in 


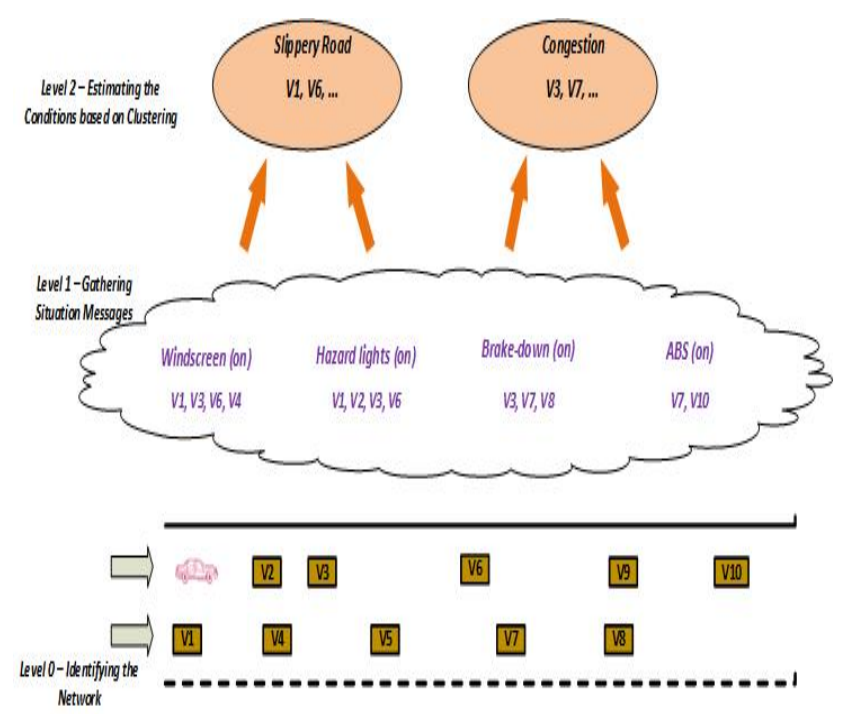

Figure 3: Sample of the Traffic Scenario

figure 3 for example, cluster of hazard lights and other cluster of windscreen wipers (on) may merge and indicate to a higher and a bigger new cluster of slippery road condition. Equations 5 and 6 identify the higher level of clusters, where $x$ and $y$ are detected traffic conditions.

$$
\begin{aligned}
& x=A \cup B \quad \text {, where } A \cap B \neq \phi \\
& y=C \cup D \quad \text {, where } \quad C \cap D \neq \phi
\end{aligned}
$$

Figure 4 illustrates how sets of traffic situations can create sets of traffic conditions. It is shown that one of sets of traffic situations may participate in more than one cluster of traffic condition decision.

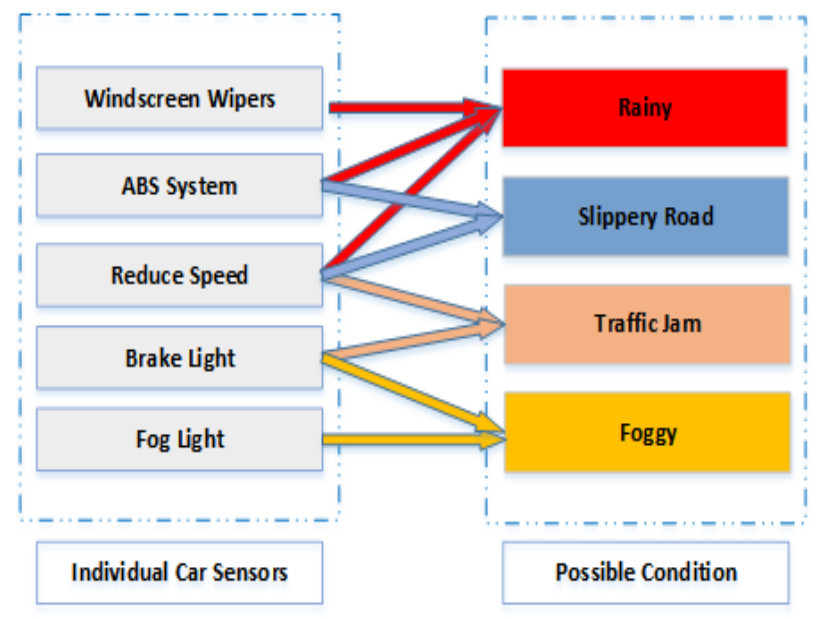

Figure 4: Example of Studying Correllations Between Set of Traffic Situations

\section{CONCLUSION}

It is widely clear that the coming intelligent transportation systems can go further from present capabilities. In this paper we have proposed a system to monitor and detect the traffic conditions based on clustering of individual cars situations.

The paper illustrated the new technique of the clustering and it also explained how to obtain the correlation among sets of situations to detect the traffic conditions. The future work will focus on implementing the new model on NS-3. In addition, the model will be implemented with different routing protocols based on IEEE 1609.4 and IEEE 802.11p standard. Results will be presented and studied based on how the model can affect on the performance of the routing protocols.

\section{REFERENCES}

Bishop, R. (2005), Intelligent vehicle technology and trends, Artech Housse, Boston, USA.

Gamati, E. A., Peytchev, E. \& Germon, R. (2011), 'Traffic condition detection algorithm (tcda) for vanet nodes in wireless intelligent transportation information systems'.

Khan, S. (2010), 'Multi constraint quality of service (qos) routing for vehicular ad-hoc networks'.

Krajzewicz, D., Erdmann, J., Behrisch, M. \& Bieker, L. (2012), 'Recent development and applications of sumosimulation of urban mobility', International Journal On Advances in Systems and Measurements 5(3 and 4), 128-138.

Li, D., Li, Q. \& Wang, J. (2012), 'Traffic information collecting algorithms for road selection decision support in vehicle ad hoc networks', International Journal of Simulation and Process Modelling 7(1), 50-56.

Llorca, D. F., Sotelo, M., Snchez, S., Ocaa, M., RodrguezAscariz, J. \& Garca-Garrido, M. (2010), 'Traffic data collection for floating car data enhancement in v2i networks', EURASIP Journal on Advances in Signal Processing 2010, 5.

Manvi, S., Kakkasageri, M. \& Pitt, J. (2009), 'Multiagent based information dissemination in vehicular ad hoc networks', Mobile information systems 5(4), 363-389.

Perkins, C., Belding-Royer, E., Das, S. et al. (2003), 'Rfc 3561-ad hoc on-demand distance vector (aodv) routing', Internet RFCs pp. 1-38.

Praba, V. L. \& Ranichitra, A. (2013), 'Detecting malicious vehicles and regulating traffic in vanet using raodv protocol'.

SUMO (2015), 'simulation of urban mobility'.

URL: Available: http://sumo.sourceforge.net

Thomas, M., Peytchev, E. \& Al-Dabass, D. (2004), Auto-sensing and distribution of traffic information in vehicular ad hoc networks, in 'Proceedings of the United Kingdom Simulation Society Conference', pp. 124-128. 
Zhao, J. \& Cao, G. (2008), 'Vadd: Vehicle-assisted data delivery in vehicular; emphasis emphasistype=', Vehicular Technology, IEEE Transactions on 57(3), 1910 1922.

Zhou, L., Cui, G., Liu, H., Wu, Z. \& Luo, D. (2010), 'Nppb: A broadcast scheme in dense vanets', Information Technology Journal 9(2), 247-256.

\section{AUTHOR BIOGRAPHIES}

DR. EVTIM PEYTCHEV is

Reader in Wireless, Mobile and Pervasive Computing in the school of Science and Technology at Nottingham Trent University, UK. He is leading the Intelligent Simulation, Modelling and Networking Research

Group, which consists of 5 lecturers, 3 Research Fellows and 6 research students. He is the Module Leader for Systems Software; and Wireless and Mobile Communications. He also teaches on the modules Software Design and Implementation; Mobile Networking; Enterprise Computing; and Computer Architecture.

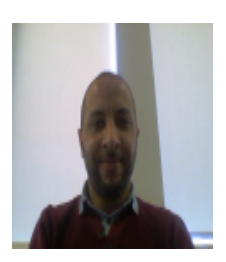

AYMAN ABUFANAS went to the College of Industry and Technology (Libya) where he received his BSc degree in electronic engineering. He worked as a technical manager in private section before moving to the High Vocational Institute for teaching. Then he went to Nottingham Trent University (the United Kingdom) where he has obtain MSc in computer and technology. Currently, he is a $\mathrm{PhD}$ student in Nottingham Trent university in the field of wireless intelligent systems. 